\title{
Enhanced Controlled Transdermal Delivery of Hydrochlorothiazide from an Ethylene-vinyl Acetate Matrix
}

\author{
Dal-Keun Kim, Jung-Chan Park, Ik-Hyun Chang, Chung Kang, Sang-Rok Ryu and Sang-Chul Shin ${ }^{\dagger}$ \\ College of Pharmacy, Chonnam National University, Gwangju 500-757, Korea \\ (Received April 21, $2010 \cdot$ Revised May 26, $2010 \cdot$ Accepted May 27, 2010)
}

\begin{abstract}
Repeated oral administration of hydrochlorothiazide, a loop diuretic, due to transient high blood levels, may cause adverse effects such as gastric disturbance, nausea, high blood sugar, and hyper lipidemia. Transdermal administration could avoid some of these systemic side effects and gastric disorders. We have developed a matrix using ethylene-vinyl acetate (EVA), a heat-processible and flexible material, for transdermal delivery of hydrochlorothiazide. Drug solubility was highest at $40 \%$ PEG-400 volume fraction. Drug release increased as concentration increased with a linear relationship between the release rate and the square root of loading dose. Increasing temperature increased drug release from the EVA matrix. The activation energy, measured from the slope of $\log \mathrm{P}$ versus $1000 / \mathrm{T}$, was $11.9 \mathrm{kcal} / \mathrm{mol}$ for a $2.5 \%$ loading dose from EVA matrix. Diethyl phthalate had the highest plasticizing effects on the release of hydrochlorothiazide. To increase the skin permeation of hydrochlorothiazide from the EVA matrix, enhancers such as the saturated fatty acids, the unsaturated fatty acids, and the non-ionic surfactants were added to the EVA matrix, and skin permeation was evaluated using a modified Keshary-Chien diffusion cell fitted with intact excised rat skin. Polyoxyethylene 23-lauryl ether showed the highest enhancing effects. In conclusion, transdermal delivery of hydrochlorothiazide could be improved from an EVA matrix containing plasticizer and permeation enhancer.
\end{abstract}

Key words - Hydrochlorothiazide, Ethylene-vinyl acetate, Controlled transdermal delivery, Matrix, Plasticizer, Permeation enhancer.

Hydrochlorothiazide, a first line thiazide diuretic, is used to treat hypertension, congestive heart failure, and symptomatic edema. However, repeated oral administration of hydrochlorothiazide, due to transient high blood levels, may cause adverse effects such as gastric disturbance, nausea, high blood sugar, and hyper lipidemia. Transdermal delivery could avoid these systemic side effects and gastric disorders after oral administration. The skin is an attractive route for drug administration because it can avoid the first-pass hepatic metabolism of drugs intended for systemic action thereby offering potentially lower drug doses and reduced side effects. Transdermal delivery circumvents the variables that could influence gastrointestinal absorption such as $\mathrm{pH}$, food intake, and gastro-intestinal motility. It also circumvents the hepatic metabolism and is therefore suitable for drugs with a low bioavailability. Transdermal drug delivery can give a constant, controlled drug input, decreasing the variations in drug plasma levels and reducing side effects particularly of drugs with a narrow therapeutic window. However, few drugs can be administered this way due to the barrier function of the skin. The stratum cor-

Corresponding Author:

Tel : +82-62-530-2924, E-mail : shinsc@chonnam.ac.kr DOI : 10.4333/KPS.2010.40.3.167 neum, the outermost layer of the skin, is the primary skin barrier, with a structure like a brick wall: the corneocytes are bricks surrounded by the mortar of the intercellular lipid lamellae. The highly organized crystalline lipid lamellae play an essential role in the barrier properties of the stratum corneum.

Many techniques can disrupt and weaken the highly organized intercellular lipids to enhance drug transport across the intact skin or to increase the driving force for drug permeation. One of the most controversial methods is the use of vesicle formulations as skin delivery systems (Loan and Joke, 2005). Penetration enhancers can also increase the permeability of the stratum corneum (Recta et al., 2005; Zrinka et al., 2008). Some of the intrinsic ingredients in these systems, such as fatty acids, phospholipids, and surfactants, enhance penetration through the skin, increasing absorption of the drug. Many new penetration enhancers have been developed to improve percutaneous absorption of drug (Amit et al., 2007). Ethylene vinyl acetate (EVA) copolymer, a member of the polyolefin family derived from random copolymerization of vinyl acetate (VA) and ethylene, has a wide range of potential applications (Suat et al., 2008).

We therefore tested whether an EVA matrix system could improve the transdermal delivery of hydrochlorothiazide. We performed release studies according to loading dose, tem- 
perature, and plasticizer content. To increase skin permeation, we optimized use of a penetration enhancer in the EVA matrix and measured drug permeation across rat skin. The objective of this study was to determine the feasibility of transdermal delivery of hydrochlorothiazide by studying its in vitro release.

\section{Materials and Methods}

\section{Materials}

Hydrochlorothiazide was supplied by Yuhan Pharm. Co., Ltd. (Korea). EVA (Ethylene vinyl acetate) was purchased from Aldrich Chemical Co., Inc. (USA). Acetyl tributyl citrate (ATBC), tributyl citrate (TBC), acetyl triethyl citrate (ATEC), and triethyl citrate (TEC) were purchased from Morflex, Inc. (USA). Diethyl phthalate (DEP) and di-n-butyl phthalate (DBP) were from Junsei Chemical Co., Ltd. (Japan). Lauric acid, oleic acid, and caprylic acid were purchased from Tokyo Kasei Kogyo Co., Ltd. 2-pyrrolidone and 1-methyl-2-pyrrolidone were purchased from Acros organics (USA). Polyoxyethylene-2-stearyl ether (Brij 72), polyoxyethylene-23lauryl ether (Brij 35) and polyoxyethylene-2-oleyl ether (Brij 92) were purchased from Sigma-Aldrich Co. (USA). Macrogol-6-glycerides, caprylocaproyl macrogol-8-glycerides, propylene glycol laurate, and propylene glycol monolaurate were gift from Gattefose (France). Stearic acid, palmitic acid, myristic acid, and linoleic acid were purchased from Kanto Chemical Co. Inc. (Japan). Acetonitrile and methanol were HPLC grade from J. T. Baker Inc. (USA). All reagents were of analytical grade and were used without further purification.

\section{Determination of Drug Solubility}

Excess amounts of hydrochlorothiazide were equilibrated with saline containing various percentage of PEG 400. Each solution was shaken at $37^{\circ} \mathrm{C}$ for $24 \mathrm{~h}$ in a shaking incubator. The solutions were then filtered through a $0.45 \mu \mathrm{m}$ filter membrane. The concentration of hydrochlorothiazide was determined at $272 \mathrm{~nm}$ by UV spectrophotometer.

\section{HPLC Determination of Hydrochlorothiazide}

Hydrochlorothiazide was assayed by HPLC methods. The HPLC system consisted of a pump (Knauer, DE/K-120, USA), and ultraviolet detector (Waters 484, USA), $\mathrm{C}_{18}$ column ( $250 \mathrm{x}$ $4.6 \mathrm{~nm}, 5 \mu \mathrm{m}$ ), degaser, and an integrator (D520A, Youngin Scientific Co., Ltd., Korea). The mobile phase was a combination of acetonitrile: water (10:90, v/v) and column temperature was maintained at ambient temperature. A flow rate of $1.0 \mathrm{~mL} / \mathrm{min}$ yielded an operation pressure of $\sim 1000 \mathrm{psi}$. The UV detector was operated at the wavelength of $272 \mathrm{~nm}$. Under these conditions, a hydrochlorothiazide peak appeared at the retention time of $4.9 \mathrm{~min}$. The detection limit for hydrochlorothiazide was $0.1 \mu \mathrm{g} / \mathrm{mL}$. A good correlation was obtained without the need for an international standard.

\section{Preparation of Hydrochlorothiazide-EVA Matrix}

Drug-containing an EVA matrix membrane was prepared by a casting process. About $2 \mathrm{~g}$ of EVA copolymer beads were dissolved in $20 \mathrm{~mL}$ of chloroform in a glass beaker. Drug was dissolved in $4 \mathrm{~mL}$ of methanol and drug solution added. This mixture was poured onto a glass plate and the solvent was allowed to evaporate off at room temperature overnight for $24 \mathrm{~h}$. The matrix was removed from the plate and dried at room temperature.

\section{In Vitro Drug Release from the EVA Matrix}

The in vitro release of hydrochlorothiazide from the EVA matrix was examined by using the modified Keshary-Chien cell. A unit of EVA matrix was clamped between the cell cap and the receptor cell. The diameter of the cell was $2 \mathrm{~cm}$ providing $3.14 \mathrm{~cm}^{2}$ effective constant areas between the matrix and the bulk solution of $21 \mathrm{~mL}$. PEG 400 solution was used as receptor solution. The receptor was maintained to $37^{\circ} \mathrm{C}$ with circulating water jacket and stirred constantly at $400 \mathrm{rpm}$. Before the experiment, the system was tested to remove the remaining air bubble in receptor site. At predetermined time intervals, the whole solution from the receptor cell was taken and replaced with fresh solution. The cumulative amount of hydrochlorothiazide released from the matrix was determined by UV spectrophotometer at $272 \mathrm{~nm}$. The effects of drug concentration on its release from the EVA matrix was studied according to drug concentration of $1 \%, 1.5 \%, 2 \%, 2.5 \%$ and $3 \%(\mathrm{w} / \mathrm{w})$. The effect of temperature on drug release was studied at $27^{\circ} \mathrm{C}, 32^{\circ} \mathrm{C}, 37^{\circ} \mathrm{C}$ and $42^{\circ} \mathrm{C}$. Each data point represents the average of three determinations.

\section{Release of Hydrochlorothiazide from the EVA Matrix}

Drug release profiles of matrix-type drug delivery systems can be represented by the Higuchi's equation (6). The release from a planar system having dispersed drug in a homogeneous matrix should follow the relationship:

$$
\mathrm{Q}=\left[\mathrm{D}\left(2 \mathrm{~A}-\mathrm{C}_{\mathrm{s}}\right) \mathrm{C}_{\mathrm{s}} \mathrm{t}\right]^{1 / 2}
$$

where $Q$ is the amount of drug released after time $t$ per unit exposed area, D is the diffusivity of the drug in the matrix, A is the initial drug concentration, and $\mathrm{C}_{\mathrm{s}}$ is the drug solubility in the matrix, He later derived a similar relationship for planar 
release from a granular matrix system in which diffusion occurs through channels (Higuchi 1963; Higuchi, 1961):

$$
\mathrm{Q}=\left[\mathrm{D} / \tau\left(2 \mathrm{~A}-\varepsilon \mathrm{C}_{\mathrm{s}}\right) \mathrm{C}_{\mathrm{s}} \mathrm{t}\right]^{1 / 2}
$$

where D and Cs refer to diffusivity and solubility in the permeability field, respectively; $\tau$ is the tortuosity of the matrix and $\varepsilon$ is the porosity of the matrix. Although the two equations are for different mechanisms, they both describe drug release as being linear with the square root of time (8):

$$
\mathrm{Q}=\mathrm{K}_{\mathrm{H}} \cdot \mathrm{t}^{1 / 2}
$$

where for the homogeneous matrix system:

$$
\mathrm{K}_{\mathrm{H}}=\left[\mathrm{D}\left(2 \mathrm{~A}-\mathrm{C}_{\mathrm{s}}\right) \mathrm{C}_{\mathrm{s}}\right]^{1 / 2}
$$

and for the granular matrix system

$$
\mathrm{K}_{\mathrm{H}}=\left[\mathrm{D} / \tau\left(2 \mathrm{~A}-\varepsilon \mathrm{C}_{\mathrm{s}}\right) \mathrm{C}_{\mathrm{s}}\right]^{1 / 2}
$$

The validity of the relationships has been confirmed experimentally by a number of workers using various systems. Singh et al., 1967; Farhadieh et al., 1971)

Preparation of Hydrochlorothiazide-EVA Matrix containing Plasticizer

A plasticizer reduces the brittleness, improves flow, and imparts flexibility, increases toughness, strength, tear resistance, and impacts resistance of the polymer. Increasing the amount of plasticizer could increase free-form elongation and decrease tensile strength and Young's modulus. Plasticizer was dropped into drug-containing EVA solution and mixed for $1 \mathrm{~h}$. This method was chosen to produce large unharmed pieces of the membrane with no molecular orientation. This mixture was poured onto a glass plate and the solvent was allowed to evaporate off at room temperature overnight. Plasticizers were added in ratios of $5 \%(\mathrm{w} / \mathrm{w})$ of the EVA matrix. The plasticizers used were the alkyl citrates such as acetyl tributyl citrate $(\mathrm{ATBC})$, tributyl citrate (TBC), acetyl triethyl citrate (ATEC), triethyl citrate (TEC). The phthalates such as diethyl phthalate (DEP) and di-n-butyl phthalate (DBP) were also used as plasticizers.

\section{Skin Preparation}

A male rat (Sprague Dawley rat strain) was sacrificed by excess etherization. The hair of the abdominal area was carefully removed with an electric clipper. A square section of the abdominal skin was excised. After incision, the adhering fat and other visceral debris in the skin were carefully removed from the undersurface with tweezers (Durrhein et al., 1980). The excised skin was used immediately.

\section{Preparation of Hydrochlorothiazide-EVA Matrix containing Enhanacer}

Enhancers could affect fluidity of the stratum corneum structure and allow better permeation. Enhancer-containing matrix was prepared by a casting process. About $2 \mathrm{~g}$ of EVA copolymer beads was dissolved in $20 \mathrm{~mL}$ of chloroform in a glass beaker. Drug was dissolved in $4 \mathrm{~mL}$ of methanol, and enhancer (5\%) was added. We used propylene glycols such as propylene glycol monolaurate, propylene glycol laurate, propylene glycol monocaprylate, and the non-ionic surfactants such as polyoxyethylene-2-oleyl ether, polyoxyethylene-2-stearyl ether, and polyoxyethylene-23-lauryl ether. Saturated fatty acids such as capric acid, myristic acid, lauric acid and the unsaturated fatty acid such as oleic acid, linoleic acid were also used. The pyrrolidones such as 2-pyrrolidone, N-methyl-2-pyrrolidone, polyvinylpyrrolidone and the glycerides such as oleyl macrogol-6-glycerides, caprylocaproyl macrogol-8-glycerides were also used. This polymer solution was poured onto a glass plate casting process and the solvent was allowed to evaporate off at room temperature for $24 \mathrm{~h}$. The matrix was removed from the plate and dried at room temperature.

\section{Permeation of Hydrochlorothiazide from the EVA Matrix containing an Enhancer through the Skin}

The permeation of hydrochlorothiazide from the EVA matrix through rat skin was examined by using the modified KesharyChien cell. The freshly excised full-thickness skin was mounted on the receptor site of the diffusion cell with the stratum corneum side facing upwards into the donor compartment and the dermal side facing downwards into the receptor compartment. Appropriate size of the matrix was placed on the stratum corneum side and covered with round glass plate and clamped tightly. Receptor medium was $40 \%$ PEG 400 solution to achieve sink condition and maintained $37^{\circ} \mathrm{C}$ by a circulating water bath. Total samples were withdrawn at predetermined time intervals and immediately replaced by an equal volume of fresh medium. The amount of drug permeated was determined at $272 \mathrm{~nm}$ by high performance liquid chromatography. Each data point represents the average of three determinations.

\section{Calculations}

The permeation rate was calculated from the slope of the linear region of the permeation profile. The flux was calculated from the slope of the linear region of the $Q$ versus $t$ permeation profile. The cumulative amount of hydrochlorothiazide through the rat skin was plotted against time $(t)$. A linear profile was observed for $12 \mathrm{~h}$ and the slope of the linear portion of the curve was determined by linear regression. 
The enhancement factor (EF) was calculated using the following equation:

$\mathrm{EF}=$ (flux of EVA matrix containing enhancer) / (flux of control sample)

\section{Results and Discussion}

\section{Solubility of Hydrochlorothiazide}

PEG 400, a hydrophilic polymer, improved the low aqueous

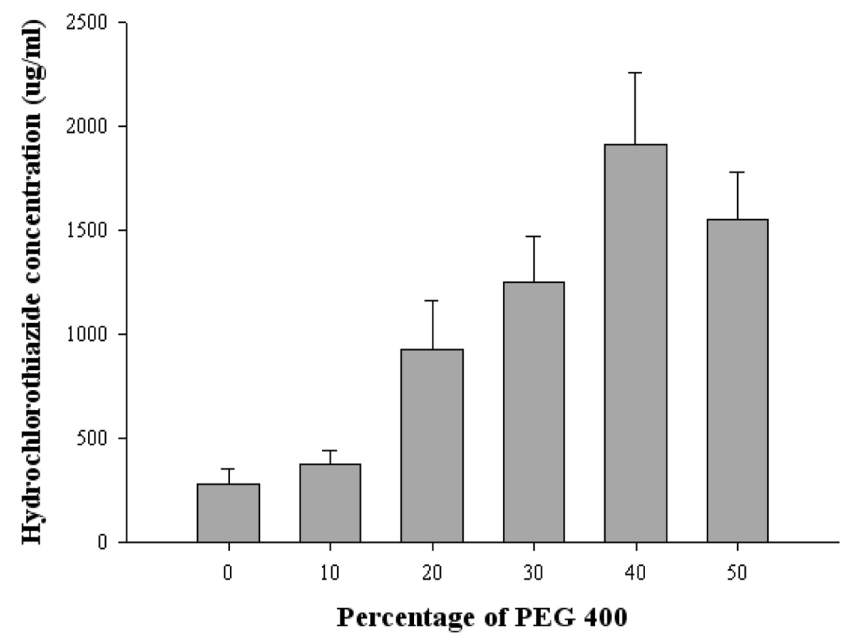

Figure 1. Solubility of hydrochlorothiazide in PEG 400.

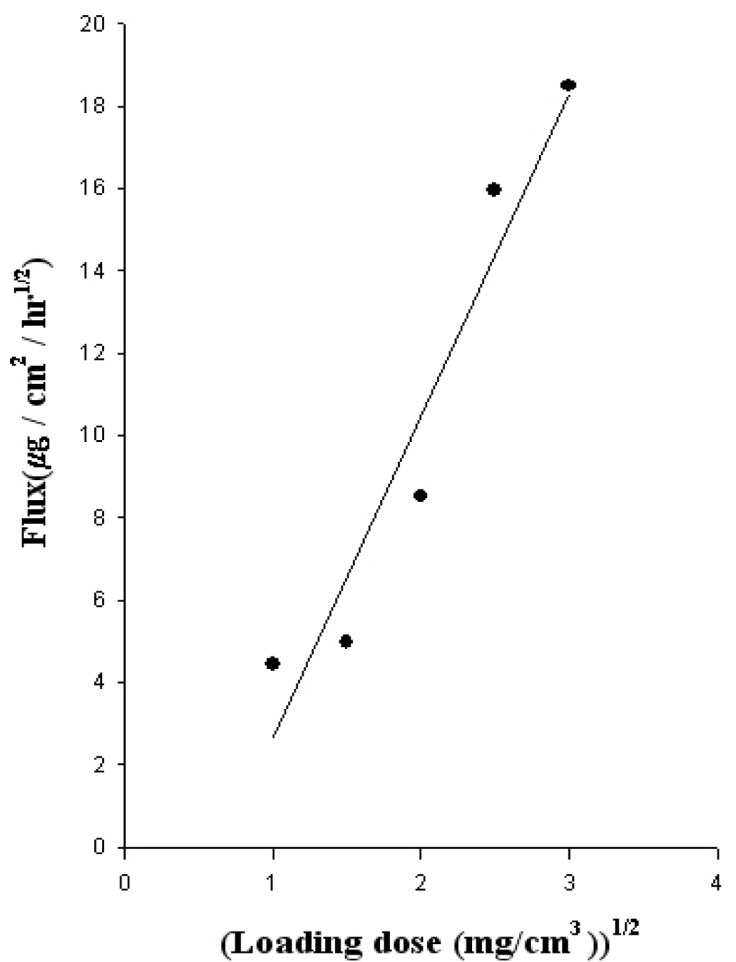

Figure 2. Relationship between hydrochlorothiazide flux and loading dose in the EVA matrix at $37^{\circ} \mathrm{C}$. solubility of hydrochlorothiazide. PEG 400 is an excellent solubilizer for many steroids (Chien and Lambert, 1975). Increasing volume fractions of PEG 400 improved the solubility of hydrochlorothiazide, with highest solubility at 40\% PEG 400 (Fig. 1).

\section{Effect of Drug Loading Dose}

The cumulative amount of hydrochlorothiazide released (Q) versus the square root of time $\left(\mathrm{t}^{1 / 2}\right)$ plot shows a good linearity for all five different concentrations. As expected from Equation 3, a plot of $\mathrm{Q} / \mathrm{t}^{1 / 2}$ versus square root of loading dose (A) yields straight line. The $\mathrm{Q} / \mathrm{t}^{1 / 2}$ increased directly proportional to the increase on the loading dose of hydrochlorothiazide (Fig. 2).

\section{Effect of Release Media Temperature}

After an initial period, drug release was approximately linear with respect to $t^{1 / 2}$. The steady-state rate of drug release $\left(\mathrm{Q} / \mathrm{t}^{1 / 2}\right)$ was estimated from the slope of the linear $\mathrm{Q}-\mathrm{t}^{1 / 2}$ profile from 0 to $12 \mathrm{~h}$. The rate of drug release increased about 1.65 fold when system temperature was raised from $27^{\circ} \mathrm{C}$ to $42^{\circ} \mathrm{C}$, but $37^{\circ} \mathrm{C}$ was chosen to reflect the temperature of the stratum corneum (Chien and Lau, 1976). Thus, the release of hydrochlorothiazide from the EVA matrix is an energy-linked

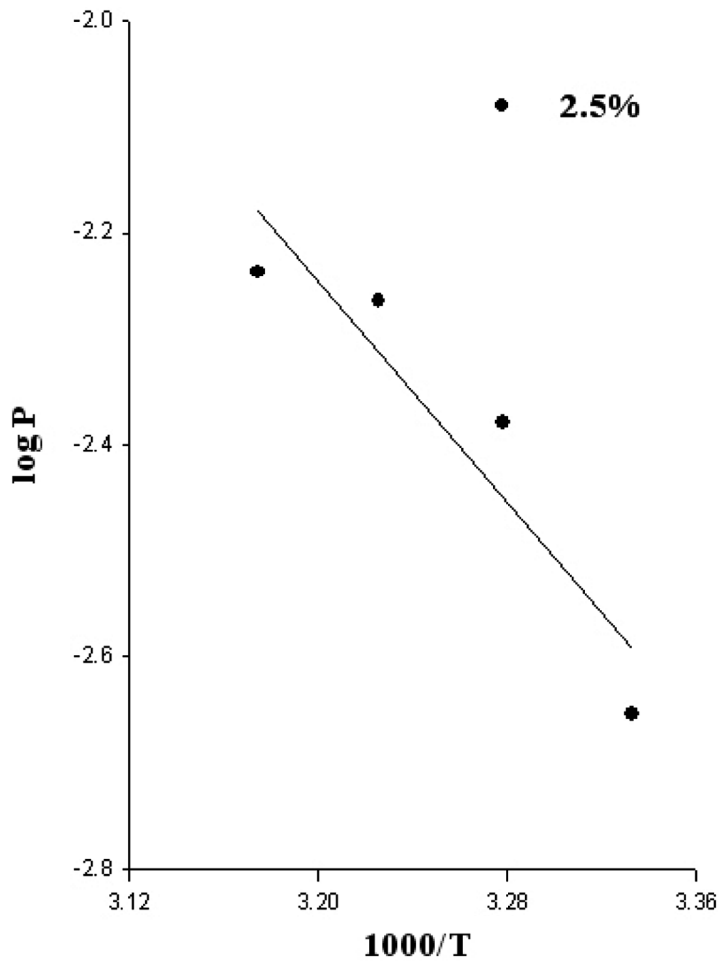

Figure 3. Effects of temperature on hydrochlorothiazide release from the EVA matrix. 
process. Furthermore, characteristics of the copolymer could change over a range of body temperatures, and special precautions for monitoring body temperature are needed in practical applications. The permeability coefficient is then defined by:

$$
\begin{aligned}
& \mathrm{P}=\text { flux/solubility } \\
& \mathrm{P}=\mathrm{P}_{0} \times \mathrm{e}^{-\mathrm{Ea} / \mathrm{RT}} \\
& \log \mathrm{P}=\log \mathrm{P}_{0}-\mathrm{E}_{\mathrm{a}} / \mathrm{R} / 2.303 / 1000 \times 1000 / \mathrm{T} \\
& \text { Slope }=-\mathrm{E}_{\mathrm{a}} / \mathrm{R} / 2.303 \times 1 / 1000 \\
& \mathrm{E}_{\mathrm{a}}=- \text { slope } \times \mathrm{R} \times 2.303 \times 1000 \mathrm{cal}
\end{aligned}
$$

As expected from Equation 8, a plot of $\log \mathrm{P}$ versus 1000/ $\mathrm{T}$ yields a straight line (Fig 3 ). The activation energy $\left(E_{a}\right)$ for drug permeation, which was measured from the slope of $\log \mathrm{P}$ versus 1000/T plot (Equation 10), was $11.9 \mathrm{kcal} / \mathrm{mol}$.

\section{Effect of Plasticizer on Drug Release}

Generally plasticizers increase the amount of drug release by increasing chain mobility of the polymer. The plasticizer will interpose itself between the polymer chains and interact with the forces held together by extending and softening the polymer matrix (Entwistle and Rowe, 1979). The plasticizer reduces the brittleness, improves flow, imparts flexibility, and increases toughness, strength, tear resistance, and impact resistance of the polymer. Plasticizer selection and concentration influences the mechanical properties and drug permeability. Increasing the amount of plasticizer could lead to an increase in free film elongation and a decrease in tensile strength. A strong interaction between a drug and a polymer has been reported to significantly influence drug release through a polymeric film (Jenquin et al., 1990). The release profiles of hydrochlorothiazide from EVA matrix containing citrate group or phthalate group as the plasticizer were shown in Table I, respectively. In the case of citrate group, there was a little

Table I. Effect of the Plasticizers on the Release of Hydrochlorothiazide from the EVA Matrix at $37^{\circ} \mathrm{C}$

\begin{tabular}{ccc}
\hline \hline & Flux $\left(\mu \mathrm{g} / \mathrm{cm}^{2} / \mathrm{hr}\right)$ & Correlation coefficient \\
\hline Control & $4.62 \pm 0.64$ & 0.9849 \\
DBP & $5.37 \pm 0.65$ & 0.996 \\
DEP & $7.39 \pm 0.66$ & 0.9697 \\
ATBC & $5.33 \pm 0.54$ & 0.9954 \\
TBC & $5.45 \pm 0.54$ & 0.9942 \\
TEC & $5.50 \pm 0.54$ & 0.9941 \\
ATEC & $5.76 \pm 0.54$ & 0.9893 \\
\hline
\end{tabular}

increased release of drug. On the other hand, phthalate group showed much increased release rate. Among the plasticizers used such as the citrates or the phthalate groups, diethyl phthalate showed the highest effect on the release of hydrochlorothiazide

Effect of Enhancer on the Permeation of Hydrochlorothiazide across the Rat Skin

We next tested whether enhancers such as the saturated fatty acids, the unsaturated fatty acids, and the non-ionic surfactants, could improve drug release. Enhancers overcome the intrinsic resistance of the stratum corneum to the permeation of a drug and may also act as a solubilizer, a fluidizer, or a plasticizer. We used a modified Keshary-Chien diffusion cell fitted with rat skin to measure hydrochlorothiazide permeation.

Fatty acids can insert between the hydrophobic tails of the stratum corneum lipid bilayer, disturbing their packing, increasing their fluidity and subsequently, decreasing the diffusional resistance to permeants (Taguchi et al., 1999). Fatty acids (FAs) can interact with intercellular lipid domains, to promote the skin permeation of drugs with a wide range of polarities. Unsaturated FAs, particularly cis conformation and $\mathrm{C}_{18}$ chain lengths, are better enhancers than their saturated counterparts, promoting the permeation of flurbiprofen (Chi et al., 1995). Linear saturated fatty acids produce less disruption of the lipid packing of the stratum corneum and are less able to insert themselves into the lipid bilayers than kinked unsaturated fatty acids of high solubility (Kandimalla et al., 1999). Saturated fatty acids did not increase permeability dramatically, but lauric acid produced the highest increase in permeation rate (Table II). Unsaturated fatty acids improved permeation rates better than saturated fatty acids, with oleic acid significantly increasing the permeation rate.

Surfactants enhance the permeability of drugs (Lopez et al., 2000; Shin et al., 2001; Squillante et al., 1998) and change the permeability of biological membranes, including skin (Lopez et al., 2000) to improve drug penetration rates. Non-ionic surfactants disrupt SC layering and increases intercellular spaces (Shin et al., 2001). Brij 72 (polyoxyethylene 23-lauryl ether) showed the best enhancing effect in other experiments, but Brij 35 (polyoxyethylene 2-stearyl ether) and Brij 92 (polyoxyethylene 2-oleyl ether) also increased permeation (Table II).

Caprylocaproyl macrogol-glyceride (Labrasol) increases the passive transport of drug molecules with low toxicity and is listed as a pharmaceutical excipient in the European Pharmacopoeia. Oleyl macrogol-6 glyceride (Labrafil) improved hydrochlorothiazide permeation. Propylene glycol (PG) is a 
Table II. Enhancement Factor According to Various Enhancers

\begin{tabular}{lcc}
\hline \hline \multicolumn{1}{c}{ Enhancer } & Flux $\left(\mu \mathrm{g} / \mathrm{cm}^{2} / \mathrm{hr}\right)$ & $\mathrm{EF}$ \\
\hline control & $0.402 \pm 0.07$ & 1.00 \\
polyoxyethylene 23-lauryl ether & $1.601 \pm 0.04$ & 3.11 \\
polyoxyethylene 2-stearyl ether & $0.656 \pm 0.01$ & 1.28 \\
polyoxyethylene 2-oleyl ether & $0.850 \pm 0.13$ & 1.66 \\
oleic acid & $0.861 \pm 0.04$ & 2.20 \\
linoleic acid & $0.655 \pm 0.07$ & 1.68 \\
caprylic acid & $0.641 \pm 0.08$ & 1.86 \\
lauric acid & $0.797 \pm 0.04$ & 2.32 \\
myristic acid & $0.478 \pm 0.05$ & 1.39 \\
oleoyl macrogol-6 glycerides & $0.677 \pm 0.01$ & 1.68 \\
caprylocaproyl macrogol-8 glycerides & $0.669 \pm 0.07$ & 1.66 \\
propylene glycol mono caprylate & $1.372 \pm 0.07$ & 2.67 \\
propylene glycol laurate & $1.429 \pm 0.11$ & 2.77 \\
propylene glycol mono laurate & $0.558 \pm 0.02$ & 1.08 \\
N-methyl pyrrolidone & $0.498 \pm 0.01$ & 2.87 \\
2-pyrrolidone & $0.310 \pm 0.01$ & 1.79 \\
Polyvinyl pyrrolidone & $0.228 \pm 0.01$ & 1.31 \\
\hline
\end{tabular}

common penetration enhancer that permeates the stratum corneum and may carry drug molecules across (Squillante et al., 1998). PG permeation could alter drug thermodynamics and modify the driving force for diffusion. PG may also partition into the tissue, facilitating uptake, and potentially disturb intercellular lipid packing within the stratum corneum bilayers (Williams and Barry, 2004). Propylene glycol laurate improved hydrochlorothiazide permeation.

Pyrrolidones have been used as penetration enhancers in human skin for hydrophilic and lipophilic permeants. Pyrrolidones partition well into the stratum corneum and alter the solvent nature of the membrane to generate 'reservoirs' within skin membranes. Such a reservoir effect offers potential for sustained drug release from the stratum corneum (Jungbauer et al., 2001). Among the penetration enhancers used, polyoxyethylene 23-lauryl ether showed the best enhancing effect (Table II).

\section{Conclusions}

Increasing temperature and drug loading increased the release rate from an EVA matrix. The plasticizer diethyl phthalate showed the best improvement in the release of hydrochlorothiazide. The penetration enhancer, polyoxyethylene 23lauryl ether, showed the best enhancement of release. In con- clusion, hydrochlorothiazide transdermal delivery could be improved with an EVA matrix containing plasticizer and permeation enhancer.

\section{Acknowledgments}

This study was financially supported by Chonnam National University, 2009.

\section{References}

Amit, G., Shailaja, M., Bozena, M., Laurent, S., 2007. Application of orthogonal collocation and regression techniques for recovering parameters of a two-pathway transdermal drug-delivery model. Comput. Chem. Eng. 31, 107-120.

Chi, S.C., Park, E.S., Kim, H., 1995. Effect of penetration enhancers on flurbiprofen permeation through rat skin. Int. J. Pharm. 126, 267-274.

Chien, Y.W., Lambert, H.J., 1975. Solubilization of steroids by multiple co-solvent systems. Chem. Pharm. Bull. 23, 1085.

Chien, Y.W., Lau, E.P.K., 1976. Controlled drug release from polymeric delivery devices IV: In vitro-in vivo correlation of subcutaneous release of norgestomet from hydrophilic implants. J. Pharm. Sci. 65, 488.

Durrhein, H., Flynn, G.L., Higuchi, W.I., Behl, C.R., 1980. Permeation of hairless mouse skin I: experimental methods and comparison with human epidermis permeation by alkanols. J. Pharm. Sci. 69, 781.

Entwistle, C.A., Rowe, R.E., 1979. Plasticization of cellulose ethers used in the film coating of tablets. J. Pharm. Pharmacol. 31, 269-272.1) Loan, H.P., Joke, A.B., 2005. Vesicles as a tool for transdermal and dermal delivery. Drug Discovery Today: Tec. 2, 67-74.

Farhadieh, B., Boradkin, S., Buddenhagen, J., 1971. Drug release from methyl acrylate-methyl methacrylate copolymer matrix I : Kinetics of release. J. Pharm. Sci. 60, 209.

Higuchi, T., 1961. Rate of release of medicaments from ointment bases containing drug in suspension. J. Pharm. Sci. 50, 874.

Higuchi, T., 1963. Mechanism of sustained-action medication. Theoretical analysis of rate of release of solid drugs dispersed in solid matrices. J. Pharm. Sci. 52, 1145.

Jenquin, M.R., Liebowitx, S.M., Sarabia, R.E., McGinity, J.W., 1990. Physical and chemical factors influencing the release of drugs from acrylic resin films. J. Pharm. Sci. 79, 811-816.

Jungbauer, F.H., Coenraads, P.J., Kardaun, S.H., 2001. Toxic hygroscopic contact reaction to N-methyl-2-pyrrolidone. Contact Dermatitis 45, 303-304.

Kandimalla, K., Kanikkannan, N., Andega, S., Singh, M., 1999. Effect of fatty acids on the permeation of melatonin across rat and pig skin in-vitro and on the transepidermal water loss in rats in-vivo. J. Pharm. Pharmacol. 51, 783-790.

H.P. Loan H. P. Joke, A.B., 2005. Vesicles as a tool for transdermal 
and dermal delivery. Drug Discovery Today: Tec. 2, 67-74.

Lopez, A., Llinares, F., Cortell, C., Herraez, M., 2000. Comparative enhancer effects of span 20 with Tween 20 and Azone on the in vitro percutaneous penetration of compounds with different lipophilicities. Int. J. Pharm. 202, 133-140.

Recta, R.G., Swantrant, K.J., Manoj, V., 2005. Colloids and Surfaces B: Biointerfaces 41, 25-32.

Shin, S.C., Cho, C.W., Oh, I.J., 2001. Effects of non-ionic surfactants as permeation enhancers towards piroxicam from the poloxamer gel through rat skins. Int. J. Pharm. 222, 199-203.

Singh, P., Desai, S.J., Simonelli, A.P., Higuchi, W.I., 1967. Release rates of solid drug mixtures dispersed in inert matrices I : Noninteracting drug mixtures. J. Pharm. Sci. 56, 1542.

Squillante, E., Needham, T., Maniar, A., Kislaliglu, S., Zia, H., 1998. Codiffusion of propylene glycol and dimethyl isosorbide in hairless mouse skin. Eur. J. Pharm. Biopharm. 46, 265-271.
Suat, U., Ahmet, R.O., Jale, Y., Selhan, K., 2008. The influence of the waste ethylene vinyl acetate copolymer on the thermal degradation of the waste polypropylene. Fuel Processing Technology 89, 1201-1206.

Taguchi, K., Fukushima, S., Yamaoka, Y., Takeuchi, Y., Suzuki, M., 1999. Enhancement of propylene glycol distribution in the skin by high purity cis-unsaturated fatty acids with different alkyl chain lengths having different double bond position. Bio. Pharm. Bull. 22, 407-411.

Williams, A.C., Barry, B.W., 2004. Penetration enhancers. Advanced Drug Delivery Reviews 56, 603-618.

Zrinka, A., Sustarsi, U., Karmen, T., Marjeta, S., Julijana, K., 2008. Influence of nanosized delivery systems with benzyl nicotinate and penetration enhancers on skin oxygenation. Int. J. Pharm. $359,220-222$. 